\title{
An Iterative Method for Blind Equalization of Multiple FIR Channels
}

\author{
Fang-Biau Ueng and Shiann-Jeng Yu \\ Department of Electrical Engineering \\ National Chung-Hsing University, Taiwan \\ E_mail:fbueng@ee.nchu.edu.tw
}

\begin{abstract}
In this paper, the performance of the multiple-shift correlation (MSC) based blind channel equalizer (BCE) under channel order mismatch due to small head and tail channel coefficients is investigated. The analysis results show that the output signal-to-interference plus noise ratio (SINR) of the MSC-based BCE is degraded due to the small channel coefficients. The performance degradation is function of the optimal output SINR, the optimal output power, and the control vector. In such channel order mismatch environment, this paper proposes a simple but effective iterative method to improve the performance. Analysis of the iterative method is also performed. Simulation examples are demonstrated to show the effectiveness of the proposed method and the analyses.
\end{abstract}

\section{Introduction}

Blind channel equalizers (BCE) without training data available receive much attention in recent years [1]-[14]. Early blind equalization techniques [1] [2] exploited the higher order statistics (HOS) of the output to identify the channels. Unfortunately, the HOS-based BCE requires a large number of data samples and huge computation load which limit their applications in fast changing environments. To circumvent the shortcomings of the HOS-based approaches, second-order-statistics (SOS) was considered in BCE. The SOS-based BCE was developed based on cyclostationary characteristics of the signal [16] [17]. The first SOS-based BCE was derived by Tong et al. [3]. They demonstrated that the SOS is sufficient for blind adaptive equalization by using fractionally sampling or using an array of sensors. Since that, extensive researches were explored in the literature. The well-known approaches are the least-squares, the subspace, and the maximum likelihood [3] [8] [9]. These blind equalizers were termed the two-step methods which estimate multiple channel parameters first and then equalize the channels based on the estimated channel parameters. However, the two-step methods are not optimal because they do not take the channel estimation error into account in the second-step optimization procedure. Recently, direct equalization estimators become more attractive [10]-[13]. The linear prediction based equalizer was developed by [13]. [12] used the adaptive beamforming technique to develop a constrained optimization method. Multiple-shift correlation (MSC) of the signals can be used in a partially adaptive channel equalizer to achieve fast convergence speed and low computation load. These direct equalizers can be adaptive, leading to much simpler realization for practical implementation.

Most of the SOS-based equalizers suffer from the performance degradation caused by the model mismatch. The mismatch may be from inadequate channel order estimation due to limited observation data or the small channel coefficients. Practical multipath channels often have small head and tail terms, selection of appropriate channel order may not be an easy task. As shown in [14] that the blind channel equalization/identification methods

This work was supported in part by the National Science Council of Taiwan under Contract NSC93-2218-E-005-003. should model only the 'significant part' of the channel composed of the 'large' channel coefficient terms. The 'small' head and/or tail terms should be neglected to avoid overmodeling the system and causing degradation of the equalization performance. [15] presented a new channel order criterion for blind equalization and [14] investigated the robustness of the LS and SS approaches by using the perturbation theory.

In this paper, we study the steady-state performance of the MSC-based equalizer. We explore the relationship between the output signal-to-interference plus noise ratio (SINR) and the small head and tail terms of the FIR channels. By applying an orthogonalization approximation to the analyses, the output SINR in terms of the small channel coefficients is derived. A degradation factor defined by the output SINR of the MSCbased equalizer over the optimal value is used to examine the performance degradation of the equalizer. We find that the degradation factor is not only a function of the small channel coefficients, but also a function of the optimal output SINR, the optimal output power, and the control vector. To reduce the degradation caused by the small channel coefficients, this paper proposes a simple iterative method. The analysis of the iterative method is also performed. From the analysis results, we identify that the iterative method indeed improves the equalization performance.

\section{The Signal Model}

Let $\mathbf{x}(\mathbf{t})=\left[x_{1}(t) x_{2}(t) \cdots x_{p}(t)\right]^{T}$ be the received data vector of an array with $p$ sensor elements, where " $T$ " represents transpose. If $\mathbf{x}(\mathbf{t})$ is sampled at the symbol rate, the digitized data vector can be written by

$$
\mathbf{x}(\mathbf{n})=\sum_{\mathbf{i}=\mathbf{0}}^{\mathbf{q}-\mathbf{1}} \mathbf{h}_{\mathbf{i}} \mathbf{s}(\mathbf{n}-\mathbf{i})+\mathbf{v}(\mathbf{n})
$$

where $\mathbf{x}(\mathbf{n})=\mathbf{x}(\mathbf{t}=\mathbf{n} \boldsymbol{\Delta} \mathbf{T})=\left[x_{1}(n) x_{2}(n) \cdots x_{p}(n)\right]^{T}$ with $\Delta T$ being the sampling interval, $\{s(n)\}$ is the input signal symbol sequence, $\mathbf{v}(\mathbf{n})=\left[v_{1}(n) v_{2}(n) \cdots v_{p}(n)\right]^{T}$ represents the additive noise, and $\left\{\mathbf{h}_{\mathbf{i}}=\left[\mathbf{h}_{\mathbf{1}}(\mathbf{i}) h_{2}(i) \cdots h_{p}(i)\right]^{T}, i=1,2, \cdots, q\right\}$ consist of all the channel parameters of the $p$ FIR channels in which the channel orders should not higher than $q-1$, but at least a channel has the channel order $q-1$.

Now consider a received data vector $\mathbf{X}(\mathbf{n})=\left[\mathbf{x}^{\mathbf{T}}(\mathbf{n}) \mathbf{x}^{\mathbf{T}}(\mathbf{n}-\mathbf{1})\right.$ $\left.\cdots \mathbf{x}^{\mathbf{T}}(\mathbf{n}-\mathbf{M}+\mathbf{1})\right]^{\mathbf{T}}$ which stacks $M$ sampled data of $\mathbf{x}(\mathbf{n})$. Using (1), $\mathbf{X}(\mathbf{n})$ can be expressed as

$$
\mathbf{X}(\mathbf{n})=\mathbf{A}(\mathbf{h}) \mathbf{S}(\mathbf{n})+\mathbf{V}(\mathbf{n})
$$

where

$\begin{aligned} \mathbf{A}(\mathbf{h}) & =\left[\begin{array}{cccccc}\mathbf{h}_{\mathbf{0}} & \mathbf{h}_{\mathbf{1}} & \cdots & \mathbf{h}_{\mathbf{q}-\mathbf{1}} & \cdots & 0 \\ \vdots & \ddots & \ddots & \ddots & \ddots & \ddots \\ 0 & \cdots & \mathbf{h}_{\mathbf{0}} & \mathbf{h}_{\mathbf{1}} & \cdots & \mathbf{h}_{\mathbf{q}-\mathbf{1}}\end{array}\right]_{(p M) \times(L)} \\ & =\left[\mathbf{A}_{\mathbf{1}}, \mathbf{A}_{\mathbf{2}}, \cdots, \mathbf{A}_{\mathbf{L}}\right]\end{aligned}$ 
is a block Toeplitz matrix with $L=q+M-1$. In (2), $\mathbf{V}(\mathbf{n})$

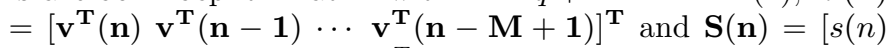
$s(n-1) \cdots s(n-L+1)]^{T}$ represents the signals with their signatures corresponding to the columns of $\mathbf{A}(\mathbf{h})$.

In this paper, $v_{i}(n)$ is assumed as the white noise with mean zero and variance $\sigma^{2}, s(n)$ is assumed to an independent identically distributed (i.i.d.) zero-mean sequence with $\mathrm{E}\left\{s(i) s^{*}(j)\right\}$ $=\delta_{\mathbf{i j}}$ where $\mathrm{E}\{\cdot\}$ is the expectation. We also assume $s(n)$ is independent to $v_{i}(n) . \mathbf{A}(\mathbf{h})$ is assumed to have full column rank. In this paper, each $\mathbf{A}_{\mathbf{i}}$ is called the direction vector corresponding to the signal $s(n-i+1)$.

Next, consider the multiple-shift correlation (MSC) matrix of $\mathbf{X}(\mathbf{n})$ defined by $\mathbf{R}_{\mathbf{X}}(\mathbf{k})=\mathrm{E}\left\{\mathbf{X}(\mathbf{n}) \mathbf{X}^{\mathbf{H}}(\mathbf{n}-\mathbf{k})\right\}$ where ' $H$ ' represents transpose and complex conjugate. Using (2), we have

$$
\mathbf{R}_{\mathbf{X}}(\mathbf{k})=\mathbf{R}_{\mathbf{S}}(\mathbf{k})+\mathbf{R}_{\mathbf{V}}(\mathbf{k})
$$

where $\mathbf{R}_{\mathbf{S}}(\mathbf{k})=\mathbf{A}(\mathbf{h}) \mathrm{E}\left\{\mathbf{S}(\mathbf{n}) \mathbf{S}^{\mathbf{H}}(\mathbf{n}-\mathbf{k})\right\} \mathbf{A}^{\mathbf{H}}(\mathbf{h})$ and $\mathbf{R}_{\mathbf{V}}(\mathbf{k})=$ $\mathrm{E}\left\{\mathbf{V}(\mathbf{n}) \mathbf{V}^{\mathbf{H}}(\mathbf{n}-\mathbf{k})\right\}$. Since $s(n)$ is i.i.d and $\mathrm{E}\left\{s(i) s^{*}(j)\right\}=\boldsymbol{\delta}_{\mathbf{i j}}$, using (3) we have

$$
\mathbf{R}_{\mathbf{S}}(\mathbf{k})=\sum_{\mathbf{i}=\mathbf{1}}^{\mathbf{L}-\mathbf{k}} \mathbf{A}_{\mathbf{i}+\mathbf{k}} \mathbf{A}_{\mathbf{i}}^{\mathbf{H}}
$$

for $L \geq k \geq 0$ and $\mathbf{R}_{\mathbf{S}}(\mathbf{k})=\mathbf{0}_{(\mathbf{p M}) \times(\mathbf{p M})}$ if $k>L$. It is noted that $\mathbf{R}_{\mathbf{V}}(\mathbf{0})=\boldsymbol{\sigma}^{2} \mathbf{I}_{(\mathbf{p M}) \times(\mathbf{p M})}$ and $\mathbf{R}_{\mathbf{V}}(\mathbf{k})=\mathbf{0}_{(\mathbf{p M}) \times(\mathbf{p M})}$ if $k \geq M$. Therefore, at $k=0$,

$$
\mathbf{R}_{\mathbf{X}}(\mathbf{0})=\sum_{\mathbf{i}=\mathbf{1}}^{\mathbf{L}} \mathbf{A}_{\mathbf{i}} \mathbf{A}_{\mathbf{i}}^{\mathbf{H}}+\sigma^{2} \mathbf{I}
$$

which is called the auto-correlation matrix of $\mathbf{X}(\mathbf{n})$ and is in general of full rank. In the following, we write $\mathbf{R}_{\mathbf{X}}=\mathbf{R}_{\mathbf{X}}(\mathbf{0})$ for simplicity.

\section{The MSC-BASEd BCE}

Let the weight vector of the equalizer for resolving the signal $s(n-d+1)$ be denoted by $\mathbf{W}_{\mathbf{d}}$ and the output of the equalizer be given by $y_{d}(n)=\mathbf{W}_{\mathbf{d}}^{\mathbf{H}} \mathbf{X}(\mathbf{n})$. Then the output power can be expressed by

$$
p_{d}=\mathrm{E}\left\{\left|y_{d}(n)\right|^{2}\right\}=\mathbf{W}_{\mathbf{d}}^{\mathbf{H}} \mathbf{R}_{\mathbf{X}} \mathbf{W}_{\mathbf{d}}
$$

and the output SINR of using $\mathbf{W}_{\mathbf{d}}$ is given by

$$
\mathrm{SINR}_{d}=\frac{\mathbf{W}_{\mathbf{d}}^{\mathbf{H}} \mathbf{A}_{\mathbf{d}} \mathbf{A}_{\mathbf{d}}^{\mathbf{H}} \mathbf{W}_{\mathbf{d}}}{\mathbf{W}_{\mathbf{d}}^{\mathbf{H}}\left(\mathbf{R}_{\mathbf{X}}-\mathbf{A}_{\mathbf{d}} \mathbf{A}_{\mathbf{d}}^{\mathbf{H}}\right) \mathbf{W}_{\mathbf{d}}}
$$

where $|\cdot|^{2}$ denotes the 2 -norm. If $p M>L$ and the direction vector $\mathbf{A}_{\mathbf{d}}$ is known, the adaptive array theory [18] shows that the optimal weight vector is

$$
\mathbf{W}_{\mathrm{od}}=\mathbf{R}_{\mathbf{X}}^{-1} \mathbf{A}_{\mathbf{d}}
$$

In this paper, the scalars and vectors with the subscript ' $O$ ' represent the optimal ones. The equalizer using (9) achieves the minimum output power given by

$$
p_{\text {od }}=\mathbf{W}_{\text {od }}^{\mathbf{H}} \mathbf{R}_{\mathbf{X}} \mathbf{W}_{\text {od }}=\mathbf{A}_{\mathbf{d}}^{\mathbf{H}} \mathbf{R}_{\mathbf{X}}^{-1} \mathbf{A}_{\mathbf{d}} .
$$

By (9) and (10), the optimal output SINR can be found by

$$
\mathrm{SINR}_{o d}=\frac{\mathbf{W}_{\mathbf{o d}}^{\mathbf{H}} \mathbf{A}_{\mathbf{d}} \mathbf{A}_{\mathbf{d}}^{\mathbf{H}} \mathbf{W}_{\mathbf{o d}}}{\mathbf{W}_{\mathbf{o d}}^{\mathbf{H}}\left(\mathbf{R}_{\mathbf{X}}-\mathbf{A}_{\mathbf{d}} \mathbf{A}_{\mathbf{d}}^{\mathbf{H}}\right) \mathbf{W}_{\mathbf{o d}}}=\frac{p_{o d}}{1-p_{o d}} .
$$

It follows that

$$
p_{o d}=\frac{\operatorname{SINR}_{o d}}{1+\mathrm{SINR}_{o d}} .
$$

(12) indicates that the minimum output power is less than unity. If the optimal output SINR is very high, $1+$ SINR $_{o d} \approx$ SINR $_{o d}$ and then the minimum output power approximates unity.

Suppose that the channel order $(q-1)$ is estimated correctly by methods, from (5) $\mathbf{R}_{\mathbf{S}}(\mathbf{L}-\mathbf{1})=\mathbf{A}_{\mathbf{L}} \mathbf{A}_{\mathbf{1}}^{\mathbf{H}}$ and $\mathbf{R}_{\mathbf{V}}(\mathbf{L}-\mathbf{1})=\mathbf{0}$ if $L-1 \geq M$. Then, using (4) we have

$$
\mathbf{R}_{\mathbf{X}}(\mathbf{L}-\mathbf{1})=\mathbf{A}_{\mathbf{L}} \mathbf{A}_{\mathbf{1}}^{\mathbf{H}} .
$$

The MSC-based BCE is developed based on (13). Since $L=q+$ $M-1$, the inequality $L-1 \geq M$ is equivalent to $q-1 \geq 1$. That means the MSC-based BCE can work under the environment that the channel order should be at least one, or equivalently, at least a multipath of the signal should exist in the environment.

Based on the SCORE approach [17], the direction vectors $\mathbf{A}_{\mathbf{1}}$ and $\mathbf{A}_{\mathbf{L}}$ can be extracted by selecting two non-zero vectors $\mathbf{b}$ and $\mathbf{c}$ and computing

$$
\begin{aligned}
& \mathbf{R}_{\mathbf{X}}^{\mathbf{H}}(\mathbf{L}-\mathbf{1}) \mathbf{b}=\mathbf{A}_{\mathbf{1}}\left(\mathbf{A}_{\mathbf{L}}^{\mathbf{H}} \mathbf{b}\right) \\
& \mathbf{R}_{\mathbf{X}}(\mathbf{L}-\mathbf{1}) \mathbf{c}=\mathbf{A}_{\mathbf{L}}\left(\mathbf{A}_{\mathbf{1}}^{\mathbf{H}} \mathbf{c}\right)
\end{aligned}
$$

where $\mathbf{b}$ and $\mathbf{c}$, termed the control vectors, are chosen to satisfy $\mathbf{A}_{\mathbf{L}}^{\mathbf{H}} \mathbf{b} \neq \mathbf{0}$ and $\mathbf{A}_{\mathbf{1}}^{\mathbf{H}} \mathbf{c} \neq \mathbf{0}$. We find that (14) and (15) are equivalent to $\mathbf{A}_{\mathbf{1}}$ and $\mathbf{A}_{\mathbf{L}}$, respectively, except for a scalar, and can be used as the estimates of the direction vectors. The weight vectors of the MSC-based BCE are given by

$$
\begin{aligned}
& \mathbf{W}_{\mathbf{1}}=\mathbf{R}_{\mathbf{X}}^{-1} \mathbf{R}_{\mathbf{X}}^{\mathbf{H}}(\mathbf{L}-\mathbf{1}) \mathbf{b} \\
& \mathbf{W}_{\mathbf{L}}=\mathbf{R}_{\mathbf{X}}^{-1} \mathbf{R}_{\mathbf{X}}(\mathbf{L}-\mathbf{1}) \mathbf{c},
\end{aligned}
$$

respectively. Substituting (16) and (17) into (7), the output power of using the weight vectors $\mathbf{W}_{\mathbf{1}}$ and $\mathbf{W}_{\mathbf{L}}$ are given by

$$
\begin{aligned}
& p_{1}=\mathbf{W}_{\mathbf{1}}^{\mathbf{H}} \mathbf{R}_{\mathbf{X}} \mathbf{W}_{\mathbf{1}}=\mathbf{b}^{\mathbf{H}} \mathbf{R}_{\mathbf{X}}(\mathbf{L}-\mathbf{1}) \mathbf{R}_{\mathbf{X}}^{-\mathbf{1}} \mathbf{R}_{\mathbf{X}}^{\mathbf{H}}(\mathbf{L}-\mathbf{1}) \mathbf{b} \\
& p_{L}=\mathbf{W}_{\mathbf{L}}^{\mathbf{H}} \mathbf{R}_{\mathbf{X}} \mathbf{W}_{\mathbf{L}}=\mathbf{c}^{\mathbf{H}} \mathbf{R}_{\mathbf{X}}^{\mathbf{H}}(\mathbf{L}-\mathbf{1}) \mathbf{R}_{\mathbf{X}}^{-\mathbf{1}} \mathbf{R}_{\mathbf{X}}(\mathbf{L}-\mathbf{1}) \mathbf{c}
\end{aligned}
$$

respectively.

\section{Performance Analysis}

From the derivations of the previous section, it is clear that the MSC-based BCE should know the channel order exactly in order to select correct time-shift index $k=L-1$ of the MSC matrix for estimating the direction vectors. However, the channel order detection should depend on strength of the channel coefficients. The equalization channels often consist of small head and tail terms so that the channel order may be underestimated. As shown in [14], the small channel coefficients should be neglected to avoid overmodeling the system but these small channel coefficients will cause performance degradation of the equalization.

Suppose that the system has $m_{1}$ small heads and $m_{2}$ small tails with $m_{1}+m_{2}<q$. Then the channel coefficients $\mathbf{h}_{\mathbf{1}}, \cdots$, $\mathbf{h}_{\mathbf{m}_{\mathbf{1}}}$ and $\mathbf{h}_{\mathbf{q}-\mathbf{m}_{\mathbf{2}}+\mathbf{1}}, \cdots, \mathbf{h}_{\mathbf{q}}$ are small comparing with $\mathbf{h}_{\mathbf{m}_{\mathbf{1}}+\mathbf{1}}$, $\mathbf{h}_{\mathbf{m}_{\mathbf{1}}+\mathbf{2}}, \cdots, \mathbf{h}_{\mathbf{q}-\mathbf{m}_{\mathbf{2}}}$. According to (3), it is noted that the di-

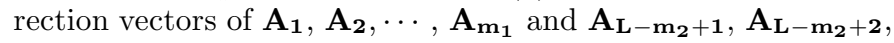
$\cdots, \mathbf{A}_{\mathbf{L}}$ will be small comparing with the direction vectors of $\mathbf{A}_{\mathbf{m}_{\mathbf{1}+\mathbf{1}}}, \mathbf{A}_{\mathbf{m}_{\mathbf{1}+\mathbf{2}}}, \cdots, \mathbf{A}_{\mathbf{L}-\mathbf{m}_{\mathbf{2}}}$. For analysis, we assume the small channel coefficients are small enough so that channel order is detected as $q-m_{1}-m_{2}-1$ rather than $q-1$. In the following, we first investigate the performance under the mismatch of the channel order due to small channel coefficients. Using (16) and (17) with $L$ now being replaced by $L-m_{1}-m_{2}$, the weight vectors of the MSC-based $\mathrm{BCE}$ can be expressed by

$$
\mathbf{W}_{\mathbf{m}_{1}+\mathbf{1}}=\mathbf{R}_{\mathbf{X}}^{-1} \mathbf{R}_{\mathbf{X}}^{\mathbf{H}}\left(\mathbf{L}-\mathbf{m}_{\mathbf{1}}-\mathbf{m}_{\mathbf{2}}-\mathbf{1}\right) \mathbf{b}
$$




$$
\mathbf{W}_{\mathbf{L}-\mathbf{m}_{2}}=\mathbf{R}_{\mathbf{X}}^{-1} \mathbf{R}_{\mathbf{X}}\left(\mathbf{L}-\mathbf{m}_{\mathbf{1}}-\mathbf{m}_{\mathbf{2}}-\mathbf{1}\right) \mathbf{c},
$$

respectively, where

$$
\begin{aligned}
& \mathbf{R}_{\mathbf{X}}\left(\mathbf{L}-\mathbf{m}_{\mathbf{1}}-\mathbf{m}_{\mathbf{2}}-\mathbf{1}\right) \\
& =\mathbf{A}_{\mathbf{L}-\mathbf{m}_{\mathbf{2}}} \mathbf{A}_{\mathbf{m}_{\mathbf{1}}+\mathbf{1}}^{\mathbf{H}}+\sum_{\mathbf{i}=\mathbf{1}, \mathbf{i} \neq \mathbf{m}_{\mathbf{1}}+\mathbf{1}}^{\mathbf{m}_{\mathbf{1}}+\mathbf{m}_{\mathbf{2}}+\mathbf{1}} \mathbf{A}_{\mathbf{L}-\mathbf{m}_{1}-\mathbf{m}_{2}-\mathbf{1}+\mathbf{i}} \mathbf{A}_{\mathbf{i}}^{\mathbf{H}}
\end{aligned}
$$

It is noted that the second term of the right side of (22) consists of the components of the small direction vectors. If their values are too small and can be negligible, (22) will reduce to $\mathbf{R}_{\mathbf{X}}(\mathbf{L}-$ $\left.\mathbf{m}_{\mathbf{1}}-\mathbf{m}_{\mathbf{2}}-\mathbf{1}\right)=\mathbf{A}_{\mathbf{L}-\mathbf{m}_{\mathbf{2}}} \mathbf{A}_{\mathbf{m}_{\mathbf{1}}+\mathbf{1}}^{\mathbf{H}}$. Therefore, the MSC-based BCE using (20) and (21) can successfully resolve the signals of $s(n-$ $\left.m_{1}\right)$ and $s\left(n-L+m_{2}+1\right)$ without significant performance degradation. This is the reason why we change the subscripts of (20) and (21).

However, in practical situation, the channel coefficients may not be small enough, the performance of the MSC-based BCE therefore degrades. It has been assumed that the direction vectors of $\mathbf{A}_{\mathbf{1}}, \mathbf{A}_{\mathbf{2}}, \cdots, \mathbf{A}_{\mathbf{m}_{\mathbf{1}}}$ and $\mathbf{A}_{\mathbf{L}}, \mathbf{A}_{\mathbf{L}-\mathbf{1}}, \cdots, \mathbf{A}_{\mathbf{L}-\mathbf{m}_{\mathbf{2}}+\mathbf{1}}$ are small comparing with the direction vectors of $\mathbf{A}_{\mathbf{m}_{\mathbf{1}}+\mathbf{1}}, \mathbf{A}_{\mathbf{m}_{\mathbf{1}}+\mathbf{2}}$, $\cdots, \mathbf{A}_{\mathbf{L}-\mathbf{m}_{\mathbf{2}}}$. The weight vectors are

$$
\begin{aligned}
& \mathbf{W}_{\mathbf{m}_{1}+\mathbf{1}}=\mathbf{R}_{\mathbf{X}}^{-1} \mathbf{R}_{\mathbf{X}}^{\mathbf{H}}\left(\mathbf{L}-\mathbf{m}_{\mathbf{1}}-\mathbf{m}_{\mathbf{2}}-\mathbf{1}\right) \mathbf{b} \\
& \mathbf{W}_{\mathbf{L}-\mathbf{m}_{\mathbf{2}}}=\mathbf{R}_{\mathbf{X}}^{-1} \mathbf{R}_{\mathbf{X}}\left(\mathbf{L}-\mathbf{m}_{\mathbf{1}}-\mathbf{m}_{\mathbf{2}}-\mathbf{1}\right) \mathbf{c}
\end{aligned}
$$

where

$$
\mathbf{R}_{\mathbf{X}}\left(\mathbf{L}-\mathbf{m}_{\mathbf{1}}-\mathbf{m}_{\mathbf{2}}-\mathbf{1}\right)=\sum_{\mathbf{i}=\mathbf{1}}^{\mathbf{m}_{1}+\mathbf{m}_{2}+\mathbf{1}} \mathbf{A}_{\mathbf{L}-\mathbf{m}_{1}-\mathbf{m}_{2}-\mathbf{1}+\mathbf{i}} \mathbf{A}_{\mathbf{i}}^{\mathbf{H}}
$$

It is noted from (25) that only $\mathbf{A}_{\mathbf{L}-\mathbf{m}_{\mathbf{2}}} \mathbf{A}_{\mathbf{m}_{\mathbf{1}+\mathbf{1}}}^{\mathbf{H}}$ is not affected by the small channel coefficients. Substituting (25) into (23) yields

$$
\mathbf{W}_{\mathbf{m}_{1}+\mathbf{1}}=\sum_{\mathbf{i}=\mathbf{1}}^{\mathbf{m}_{1}+\mathbf{m}_{2}+\mathbf{1}} \mathbf{R}_{\mathbf{X}}^{-1} \mathbf{A}_{\mathbf{i}} \mathbf{A}_{\mathbf{L}-\mathbf{m}_{1}-\mathbf{m}_{2}-\mathbf{1}+\mathbf{i}}^{\mathbf{H}} \mathbf{b}
$$

Using (26), we have

$$
\mathbf{A}_{\mathbf{m}_{1}+\mathbf{1}}^{\mathbf{H}} \mathbf{W}_{\mathbf{m}_{1}+\mathbf{1}} \approx \mathbf{p}_{\mathbf{o}\left(\mathbf{m}_{1}+\mathbf{1}\right)} \mathbf{A}_{\mathbf{L}-\mathbf{m}_{\mathbf{2}}}^{\mathbf{H}} \mathbf{b}
$$

where we have used the following approximation

$$
\mathbf{A}_{\mathbf{m}_{1}+1}^{\mathbf{H}} \mathbf{R}_{\mathbf{X}}^{-1} \mathbf{A}_{\mathbf{i}} \approx \mathbf{0} \text { for } \mathbf{i} \neq \mathbf{m}_{\mathbf{1}}+\mathbf{1}
$$

The output power of using $\mathbf{W}_{\mathbf{m}_{\mathbf{1}}+\mathbf{1}}$ is given by

$$
\mathbf{W}_{\mathbf{m}_{1}+\mathbf{1}}^{\mathbf{H}} \mathbf{R}_{\mathbf{X}} \mathbf{W}_{\mathbf{m}_{1}+\mathbf{1}} \approx \sum_{\mathbf{i}=\mathbf{1}}^{\mathbf{m}_{1}+\mathbf{m}_{2}+\mathbf{1}} \mathbf{p}_{\mathrm{oi}}\left|\mathbf{b}^{\mathbf{H}} \mathbf{A}_{\mathbf{L}-\mathbf{m}_{1}-\mathbf{m}_{2}-\mathbf{1}+\mathbf{j}}\right|^{\mathbf{2}}
$$

where we have used the approximation of (28) again. From (8), the output SINR of $\mathbf{W}_{\mathbf{m}_{\mathbf{1}}+\mathbf{1}}$ is given by

$$
\operatorname{SINR}_{m_{1}+1}=\frac{\mathbf{W}_{\mathbf{m}_{1}+\mathbf{1}}^{\mathbf{H}} \mathbf{A}_{\mathbf{m}_{\mathbf{1}}+\mathbf{1}} \mathbf{A}_{\mathbf{m}_{\mathbf{1}}+\mathbf{1}}^{\mathbf{H}} \mathbf{W}_{\mathbf{m}_{\mathbf{1}}+\mathbf{1}}}{\mathbf{W}_{\mathbf{m}_{\mathbf{1}}+\mathbf{1}}^{\mathbf{H}}\left(\mathbf{R}_{\mathbf{X}}-\mathbf{A}_{\mathbf{m}_{\mathbf{1}}+\mathbf{1}} \mathbf{A}_{\mathbf{m}_{\mathbf{1}}+\mathbf{1}}^{\mathbf{H}}\right) \mathbf{W}_{\mathbf{m}_{\mathbf{1}}+\mathbf{1}}}
$$

Substituting (27) and (29) into (30) and after some calculations, the output SINR of the equalizer using (20) can be expressed by

$$
\operatorname{SINR}_{m_{1}+1} \approx \operatorname{SINR}_{o\left(m_{1}+1\right)} F_{m_{1}+1}
$$

where $F_{m_{1}+1}$ is called the degradation factor with

$$
F_{m_{1}+1}=\left(1+\left(\frac{\operatorname{SINR}_{o\left(m_{1}+1\right)}}{p_{o\left(m_{1}+1\right)}}\right)\left(\frac{D_{m_{1}+1}}{p_{o\left(m_{1}+1\right)}\left|\mathbf{b}^{\mathbf{H}} \mathbf{A}_{\mathbf{L}-\mathbf{m}_{\mathbf{2}}}\right|^{\mathbf{2}}}\right)\right)^{-1}
$$

where

$$
D_{m_{1}+1}=\sum_{i=1, i \neq m_{1}+1}^{m_{1}+m_{2}+1} p_{o i}\left|\mathbf{b}^{\mathbf{H}} \mathbf{A}_{\mathbf{L}-\mathbf{m}_{\mathbf{1}}-\mathbf{m}_{\mathbf{2}}-\mathbf{1}+\mathbf{i}}\right|^{\mathbf{2}}
$$

By the similar way, we can derive the output SINR of the equalizer using $\mathbf{W}_{\mathbf{L}-\mathbf{m}_{\mathbf{2}}}$ by

$$
\operatorname{SINR}_{L-m_{2}} \approx \operatorname{SINR}_{o\left(L-m_{2}\right)} F_{L-m_{2}}
$$

where

$$
F_{L-m_{2}}=\left(1+\left(\frac{\operatorname{SINR}_{o\left(L-m_{2}\right)}}{p_{o\left(L-m_{2}\right)}}\right)\left(\frac{D_{L-m_{2}}}{p_{o\left(L-m_{2}\right)}\left|\mathbf{c}^{\mathbf{H}} \mathbf{A}_{\mathbf{m}_{\mathbf{1}}+\mathbf{1}}\right|^{\mathbf{2}}}\right)\right)^{-1}
$$

and

$$
D_{L-m_{2}}=\sum_{i=1, i \neq m_{1}+1}^{m_{1}+m_{2}+1} p_{o\left(L-m_{1}-m_{2}-1+i\right)}\left|\mathbf{c}^{\mathbf{H}} \mathbf{A}_{\mathbf{i}}\right|^{\mathbf{2}} .
$$

\section{The Proposed Iterative Method}

In this section, we propose a simple but effective iterative method to reduce the sensitivity from the small channel coefficients. This method was also ever used for performance improvement of the adaptive spatial filtering [19]. Unfortunately, detailed performance analysis was not given by [19].

Let $\mathbf{W}_{\mathbf{m}_{\mathbf{1}} \mathbf{1}}(\mathbf{l})$ and $\mathbf{W}_{\mathbf{L}-\mathbf{m}_{\mathbf{2}}}(\mathbf{l})$ represent the weight vectors after $l$ iterations. The iterative method is described as follows.

$$
\begin{aligned}
& \mathbf{W}_{\mathbf{m}_{1}+\mathbf{1}}(\mathbf{l})=\mathbf{R}_{\mathbf{X}}^{-1} \mathbf{R}_{\mathbf{X}}^{\mathbf{H}}\left(\mathbf{L}-\mathbf{m}_{\mathbf{1}}-\mathbf{m}_{\mathbf{2}}-\mathbf{1}\right) \mathbf{b}(\mathbf{l}) \\
& \mathbf{W}_{\mathbf{L}-\mathbf{m}_{2}}(\mathbf{l})=\mathbf{R}_{\mathbf{X}}^{-1} \mathbf{R}_{\mathbf{X}}\left(\mathbf{L}-\mathbf{m}_{\mathbf{1}}-\mathbf{m}_{\mathbf{2}}-\mathbf{1}\right) \mathbf{c}(\mathbf{l})
\end{aligned}
$$

where the ' $l$ 'th control vectors are selected by $\mathbf{b}(\mathbf{l})=$ $\mathbf{W}_{\mathbf{L}-\mathbf{m}_{\mathbf{2}}}(\mathbf{l}-\mathbf{1})$ and $\mathbf{c}(\mathbf{l})=\mathbf{W}_{\mathbf{m}_{\mathbf{1}}+\mathbf{1}}(\mathbf{l}-\mathbf{1})$ with the initial vectors $\mathbf{b}(\mathbf{1})=\mathbf{W}_{\mathbf{L}-\mathbf{m}_{\mathbf{2}}}(\mathbf{0})=\mathbf{b}$ and $\mathbf{c}(\mathbf{1})=\mathbf{W}_{\mathbf{m}_{\mathbf{1}+\mathbf{1}}}(\mathbf{0})=\mathbf{c}$. Then we have

$$
\mathbf{W}_{\mathbf{m}_{\mathbf{1}}+\mathbf{1}}(\mathbf{1})=\mathbf{R}_{\mathbf{X}}^{-\mathbf{1}} \mathbf{R}_{\mathbf{X}}^{\mathbf{H}}\left(\mathbf{L}-\mathbf{m}_{\mathbf{1}}-\mathbf{m}_{\mathbf{2}}-\mathbf{1}\right) \mathbf{b}
$$

$$
\begin{aligned}
\mathbf{W}_{\mathbf{m}_{1}+\mathbf{1}}(\mathbf{2}) & =\mathbf{R}_{\mathbf{X}}^{-1} \mathbf{R}_{\mathbf{X}}^{\mathbf{H}}\left(\mathbf{L}-\mathbf{m}_{\mathbf{1}}-\mathbf{m}_{\mathbf{2}}-\mathbf{1}\right) \mathbf{b}(\mathbf{2}) \\
& =\mathbf{R}_{\mathbf{X}}^{-1} \mathbf{R}_{\mathbf{X}}^{\mathbf{H}}\left(\mathbf{L}-\mathbf{m}_{\mathbf{1}}-\mathbf{m}_{\mathbf{2}}-\mathbf{1}\right) \mathbf{W}_{\mathbf{L}-\mathbf{m}_{\mathbf{2}}}(\mathbf{1}) \\
& =\Phi \mathbf{c}
\end{aligned}
$$

where

$$
\Phi \approx \sum_{\mathbf{i}=\mathbf{1}}^{\mathbf{m}_{1}+\mathbf{m}_{\mathbf{2}}+\mathbf{1}} \mathbf{p}_{\mathbf{o}\left(\mathbf{L}-\mathbf{m}_{1}-\mathbf{m}_{2}-\mathbf{1}+\mathbf{i}\right)} \mathbf{R}_{\mathbf{X}}^{-1} \mathbf{A}_{\mathbf{i}} \mathbf{A}_{\mathbf{i}}^{\mathbf{H}}
$$

By (39) and (40), we can find that

$$
\begin{aligned}
\mathbf{W}_{\mathbf{m}_{1}+\mathbf{1}}(\mathbf{2 l}+\mathbf{1})= & \boldsymbol{\Phi}^{1} \mathbf{R}_{\mathbf{X}}^{-1} \mathbf{R}_{\mathrm{X}}^{\mathrm{H}}\left(\mathbf{L}-\mathbf{m}_{\mathbf{1}}-\mathbf{m}_{\mathbf{2}}-\mathbf{1}\right) \mathbf{b} \\
& \mathbf{W}_{\mathbf{m}_{1}+\mathbf{1}}(\mathbf{2 l})=\boldsymbol{\Phi}^{1} \mathbf{c}
\end{aligned}
$$

Using (40), we can approximate $\boldsymbol{\Phi}^{1}$ by

$$
\Phi^{1} \approx \sum_{\mathbf{i}=1}^{\mathbf{m}_{1}+\mathbf{m}_{2}+\mathbf{1}} \mathbf{p}_{\mathrm{o}\left(\mathbf{L}-\mathbf{m}_{1}-\mathbf{m}_{2}-\mathbf{1}+\mathbf{i}\right)}^{\mathbf{l}} \mathbf{p}_{\mathrm{oi}}^{\mathbf{l}-\mathbf{1}} \mathbf{R}_{\mathrm{X}}^{-1} \mathbf{A}_{\mathbf{i}} \mathbf{A}_{\mathbf{i}}^{\mathrm{H}}
$$

Now, let us consider the odd case first. By (41) and (43),

$$
\approx \sum_{i=1}^{\mathbf{W}_{\mathbf{m}_{\mathbf{1}}+\mathbf{1}}(\mathbf{2 l}+\mathbf{1})} p_{o\left(L-m_{1}-m_{2}-1+i\right)}^{m_{1}+m_{2}+1} p_{o i}^{l} \mathbf{R}_{\mathbf{X}}^{-\mathbf{1}} \mathbf{A}_{\mathbf{i}} \mathbf{A}_{\mathbf{L}-\mathbf{m}_{\mathbf{1}}-\mathbf{m}_{\mathbf{2}}-\mathbf{1}+\mathbf{i}}^{\mathbf{H}} \mathbf{b} .
$$


Then

$$
\mathbf{A}_{\mathbf{m}_{1}+1}^{\mathbf{H}} \mathbf{W}_{\mathbf{m}_{1}+1}(2 \mathbf{l}+\mathbf{1}) \approx \mathbf{p}_{\mathrm{o}\left(\mathrm{L}-\mathrm{m}_{2}\right)}^{1} \mathbf{p}_{\mathrm{o}\left(\mathbf{m}_{1}+1\right)}^{\mathrm{l}+1} \mathbf{A}_{\mathbf{L}-\mathrm{m}_{2}}^{\mathrm{H}} \mathbf{b}
$$

and

$$
\approx \quad \sum_{i=1}^{\mathbf{W}_{\mathbf{m}_{\mathbf{1}}+\mathbf{1}}^{\mathbf{H}}(\mathbf{2 l}+\mathbf{1}) \mathbf{R}_{\mathbf{X}} \mathbf{W}_{\mathbf{m}_{\mathbf{1}}+\mathbf{1}}(\mathbf{2 l}+\mathbf{1})} p_{o\left(L-m_{1}-m_{2}-1+i\right)}^{m_{1}+m_{2}+1} p_{o i}^{2 l+1}\left|\mathbf{b}^{\mathbf{H}} \mathbf{A}_{\mathbf{L}-\mathbf{m}_{\mathbf{1}}-\mathbf{m}_{\mathbf{2}}-\mathbf{1}+\mathbf{i}}\right|^{\mathbf{2}} .
$$

Using (45) and (46), the output SINR after $(2 l+1)$ iterations can be found by

$$
\operatorname{SINR}_{m_{1}+1}(2 l+1) \approx \operatorname{SINR}_{o\left(m_{1}+1\right)} F_{m_{1}+1}(2 l+1)
$$

where

$$
\begin{aligned}
& F_{m_{1}+1}^{-1}(2 l+1)= \\
& 1+\left(\frac{\operatorname{SINR}_{o\left(m_{1}+1\right)}}{p_{o\left(m_{1}+1\right)}}\right)\left(\frac{D_{m_{1}+1}(2 l+1)}{p_{o\left(L-m_{2}\right)}^{2 l} p_{o\left(m_{1}+1\right)}^{2 l+1}\left|\mathbf{b}^{\mathbf{H}} \mathbf{A}_{\mathbf{L}-\mathbf{m}_{\mathbf{2}}}\right|^{\mathbf{2}}}\right)
\end{aligned}
$$

with

$$
=\sum_{i=1, i \neq m_{1}+1}^{D_{m_{1}+1}(2 l+1)} p_{o\left(L-m_{1}-m_{2}-1+i\right)}^{2 l} p_{o i}^{2 l+1}\left|\mathbf{b}^{\mathbf{H}} \mathbf{A}_{\mathbf{L}-\mathbf{m}_{\mathbf{1}}-\mathbf{m}_{\mathbf{2}}-\mathbf{1}+\mathbf{i}}\right|^{\mathbf{2}} .
$$

Next, consider the even case. Using (42) and (43) yields

$$
\begin{aligned}
\mathbf{A}_{\mathbf{m}_{\mathbf{1}}+\mathbf{1}}^{\mathbf{H}} \mathbf{W}_{\mathbf{m}_{\mathbf{1}}+\mathbf{1}}(\mathbf{2 l}) \approx \mathbf{p}_{\mathbf{o}\left(\mathbf{L}-\mathbf{m}_{\mathbf{2}}\right)}^{\mathbf{l}} \mathbf{p}_{\mathbf{o}\left(\mathbf{m}_{\mathbf{1}}+\mathbf{1}\right)}^{\mathbf{l}} \mathbf{A}_{\mathbf{m}_{\mathbf{1}}+\mathbf{1}}^{\mathbf{H}} \mathbf{c} \\
\approx \sum_{i=1}^{\mathbf{W}_{\mathbf{m}_{\mathbf{1}}+\mathbf{1}}^{\mathbf{H}}(\mathbf{2 l}) \mathbf{R}_{\mathbf{X}} \mathbf{W}_{\mathbf{m}_{\mathbf{1}}+\mathbf{1}}(\mathbf{2 l})} p_{o\left(L-m_{1}-m_{2}-1+i\right)}^{m_{1}+m_{2}+1} p^{2 l}\left|\mathbf{c}^{\mathbf{H}} \mathbf{A}_{\mathbf{i}}\right|^{\mathbf{2}} .
\end{aligned}
$$

As a result, the output SINR after $2 l$ iterations can be found by

$$
\operatorname{SINR}_{m_{1}+1}(2 l) \approx \operatorname{SINR}_{o\left(m_{1}+1\right)} F_{m_{1}+1}(2 l)
$$

where

$$
\begin{aligned}
& F_{m_{1}+1}^{-1}(2 l) \\
= & 1+\left(\frac{\operatorname{SINR}_{o\left(m_{1}+1\right)}}{p_{o\left(m_{1}+1\right)}}\right)\left(\frac{D_{m_{1}+1}(2 l)}{p_{o\left(L-m_{2}\right)}^{2 l} p_{o\left(m_{1}+1\right)}^{2 l-1}\left|\mathbf{c}^{\mathbf{H}} \mathbf{A}_{\mathbf{m}_{\mathbf{1}}+\mathbf{1}}\right|^{\mathbf{2}}}\right)
\end{aligned}
$$

with

$$
D_{m_{1}+1}(2 l)=\sum_{i=1, i \neq m_{1}+1}^{m_{1}+m_{2}+1} p_{o\left(L-m_{1}-m_{2}-1+i\right)}^{2 l} p_{o i}^{2 l-1}\left|\mathbf{c}^{\mathbf{H}} \mathbf{A}_{\mathbf{i}}\right|^{\mathbf{2}} .
$$

By the similar way as shown above, we can derive that

$$
\begin{aligned}
\operatorname{SINR}_{L-m_{2}}(2 l+1) & \approx \operatorname{SINR}_{o\left(L-m_{2}\right)} F_{L-m_{2}}(2 l+1) \\
\operatorname{SINR}_{L-m_{2}}(2 l) & \approx \operatorname{SINR}_{o\left(L-m_{2}\right)} F_{L-m_{2}}(2 l)
\end{aligned}
$$

where

$$
\begin{aligned}
& F_{L-m_{2}}^{-1}(2 l+1)= \\
& 1+\left(\frac{\operatorname{SINR}_{o\left(L-m_{2}\right)}}{p_{o\left(L-m_{2}\right)}}\right)\left(\frac{D_{L-m_{2}}(2 l+1)}{p_{o\left(m_{1}+1\right)}^{2 l} p_{o\left(L-m_{2}\right)}^{2 l+1} \mid \mathbf{c}^{\mathbf{H}} \mathbf{A}_{\left.\mathbf{m}_{\mathbf{1}+\mathbf{1}}\right|^{\mathbf{2}}}}\right) \\
& F_{L-m_{2}}^{-1}(2 l)= \\
& 1+\left(\frac{\operatorname{SINR}_{o\left(L-m_{2}\right)}}{p_{o\left(L-m_{2}\right)}}\right)\left(\frac{D_{L-m_{2}}(2 l)}{p_{o\left(m_{1}+1\right)}^{2 l} p_{o\left(L-m_{2}\right)}^{2 l-1}\left|\mathbf{b}^{\mathbf{H}} \mathbf{A}_{\mathbf{L}-\mathbf{m}_{\mathbf{2}}}\right|^{\mathbf{2}}}\right)
\end{aligned}
$$

with

$$
\begin{aligned}
= & \sum_{i=1, i \neq m_{1}+1}^{D_{L-m_{2}}(2 l+1)} p_{o i}^{2 l} p_{o\left(L-m_{1}-m_{2}-1+i\right)}^{2 l+1}\left|\mathbf{c}^{\mathbf{H}} \mathbf{A}_{\mathbf{i}}\right|^{\mathbf{2}} \\
= & \sum_{i=1, i \neq m_{1}+1}^{m_{L}+m_{2}+m_{2}(2 l)} p_{o i}^{2 l} p_{o\left(L-m_{1}-m_{2}-1+i\right)}^{2 l-1}\left|\mathbf{b}^{\mathbf{H}} \mathbf{A}_{\mathbf{L}-\mathbf{m}_{1}-\mathbf{m}_{2}-\mathbf{1}+\mathbf{i}}\right|^{\mathbf{2}} .
\end{aligned}
$$

\section{The Illustrated Examples}

In this section, we provide an example to verify the analysis results derived at the previous sections. An 4-element array with channel order 3 is examined. The channel parameters are present in the Table I. In the following results, we multiply $\mathbf{h}_{\mathbf{0}}$ and $\mathbf{h}_{\mathbf{3}}$ by a small scalar $\rho$ as $\rho \mathbf{h}_{\mathbf{0}}$ and $\rho \mathbf{h}_{\mathbf{3}}$ in the simulations. That is $m_{1}=m_{2}=1$ is selected to examine the effect of the small head and tail channel coefficients on the array output SINR. The control vectors are chosen as $\mathbf{b}=\mathbf{c}=[\mathbf{1}, \mathbf{1}, \cdots, \mathbf{1}]^{\mathbf{T}}$.

Figure 1 shows the values of the performance degradation at different scalar value $\rho$. In this example, the input $\mathrm{SNR}=30$ $\mathrm{dB}$. The dimension of $\mathbf{X}(\mathbf{n})$ is 24 , thus $L=q+M-1=9$, $m_{1}+1=2$, and $L-m_{2}=8$. The iterative method using 5 iterations is also performed for verification. From this figure, we find that the analyses of the equalizer without iteration quite match the actual simulation results of the MSC-based BCE. The improvement of using the iteration method is also verified. The analyses of the equalizer using five iterations is close to the simulation results.

Figure 2 shows the performance degradation versus the input SNR. In this example, the scalar $\rho=0.005$, the dimension of $\mathbf{X}(\mathbf{n})$ is 24 , and the number of iterations is 5. From this figure, we find that the analyses of the equalizer without iteration match the actual simulation results of the MSC-based BCE. The improvement of using the iteration method is quite significant at the range of $\mathrm{SNR}=20 \mathrm{~dB}$ to $\mathrm{SNR}=40 \mathrm{~dB}$.

Figure 3 shows the performance degradation versus the dimension $p M$. In this example, the scalar $\rho=0.005$, the input $\mathrm{SNR}=30 \mathrm{~dB}$, and the number of iterations is 5 . Again, we find that the analyses of the equalizer without iteration quite match the actual simulation results of the MSC-based BCE. The improvement of using the iteration method is very significant. Using 5 iterations can almost eliminate the degradation due to small channel coefficients.

Figure 4 shows the performance degradation versus the number of iterations. In this example, the scalar $\rho=0.005$, the input $\mathrm{SNR}=30 \mathrm{~dB}$, and the dimension of $\mathbf{X}(\mathbf{n})$ is 24 . From the figures, the analyses of the equalizer without iteration quite match the actual simulation results of the MSC-based BCE using $\mathbf{W}_{\mathbf{2}}$. From Figure $4, \mathbf{W}_{\mathbf{2}}$ using 3 iterations can eliminate the degradation due to small channel coefficients.

\section{REFERENCES}

[1] G. Giannakis and J. Mendel,"Identification of nonminimum phase system using higher order statistics," IEEE Trans. Acoust., Speech, Signal Processing, vol. 37, pp.360-377, Mar. 1989.

[2] B. Porat and B. Friedlander,"Blind equalization for digital communication channel using higher order moments," IEEE Trans. Signal Processing, vol. 39, pp.522-526, Feb. 1991.

[3] L. Tong, G. Xu, and T. Kailath, "Blind identification and equalization based on second-order statistics: A time-domain approach," IEEE Trans. Information Theory, vol. 40, pp.340-349, Mar. 1994.

[4] D. Slock, "Blind fractionally-spaced equalization, perfect reconstruction filterbanks, and multilinear prediction," in Proc. of Intl. Conf. Acoust. Speech, Signal Processing, Adelaide, Australia, Apr. 1994. 
(a)

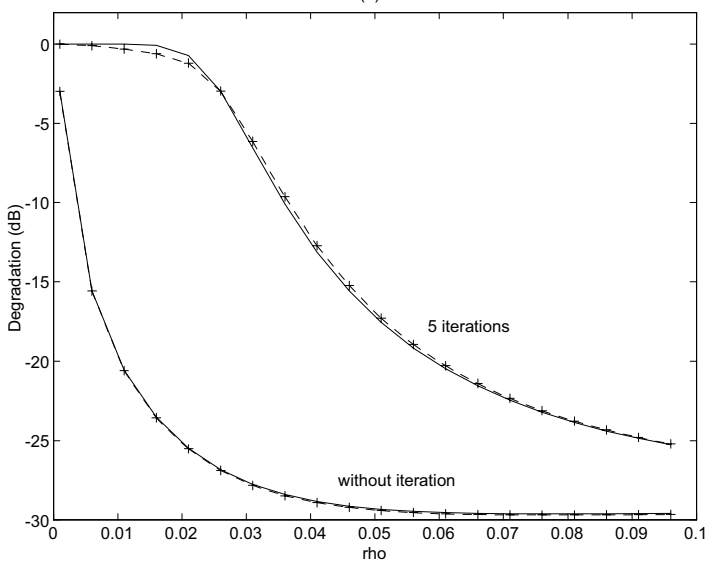

Fig. 1. The degradation factor versus $\rho$. Solid curves are the analysis results and the dash curves with ' + ' are the simulation results of the MSC-based BCE.

[5] M. Gurelli and C. Nikias, "EVAM: An eigenvector-based deconvolution of input colored signals," IEEE Trans. Signal Processing, vol. 43, pp.134-149, Jan. 1995.

[6] E. Moulines, etc., "Subspace methods for the blind identification of multichannel FIR filters," IEEE Trans. Signal Processing, vol. 43, pp.516-525, Feb. 1995.

[7] H. Liu and G. Xu, "Closed-form blind symbol estimation in digital communications," IEEE Trans. Signal Processing, vol. 43, pp.2714-2723, Nov. 1995

[8] G. Xu, etc., "A least squares approach to blind channel identification," IEEE Trans. Signal Processing, vol. 43, pp.2982-2993, Dec 1995.

[9] Y. Hua, "Fast maximum likelihood for blind identification of multiple FIR channels," IEEE Trans. Signal Processing, vol. 44, pp.661-672, Mar. 1996.

[10] G. Giannakis and S. Halford, "Blind fractionally spaced equalization of noisy FIR channels: Direct and adaptive solution," IEEE Trans. Signal Processing, vol. 45, pp.2227-2292, Sept. 1997.

[11] G. Giannakis and C. Tepedelenlioglu, "Direct blind equalizers of multiple FIR channels: A deterministic approach," IEEE Trans. Signal Processing, vol. 47 , pp.62-74, Jan. 1999

[12] M. Tsatsanis and Z. Xu, "Constrained optimization methods for direct blind equalization," IEEE Trans. Signal Processing, vol. 17, pp.424-433, Mar. 1999.

[13] J. Mannerkoski and D. Taylor, "Blind equalization using least-squares lattice prediction," IEEE Trans. Signal Processing, vol. 47, pp.630-640, Mar. 1999 .

[14] A. Liavas, et al., "Blind channel approximation: Effective channel order determination", IEEE Trans. Signal Processing, vol. 47, pp. 3336-3344, Dec. 1999.

[15] A. Liavas, et al., "Robustness of least-squares and subspace methods for blind channel identification/equalization with respect to effective channel undermodeling/overmodeling", IEEE Trans. Signal Processing, vol. 47, pp. 1636-1645, June 1999.

[16] S.V. Schell and W.A. Gardner, "Programmable Canonical Correlation Analysis: a Flexible Framework for Blind Adaptive Spatial Filtering", IEEE Trans. Signal Processing, Vol.43, pp. 2898-2908, Dec. 1995.

[17] B.G. Agee, S.V. Schell, and W.A. Gardner, "Spectral Self-Coherence Restoral: A New Approach to Blind Adaptive Extraction Using Antenna Arrays", Proc. IEEE, Vol. 78, pp. 753-766, Apr. 1990.

[18] R.T. Comption, Jr., Adaptive Antennas, Concepts, and Performance, Englewood Cliffs, NJ: Prentice Hall, 1989.

[19] M. F. Kahn, W. A. Gardner, M. A. Mow, "Programmable Canonical correlation analyzers with recursion and feedback", in Proc. Asilomar Conf. Signals Syst. Comput., pp. 351-356, 1996.

Table I The channel impulse responses

\begin{tabular}{c|c|c|c|c} 
ch. & $\mathbf{h}_{\mathbf{0}}$ & $\mathbf{h}_{\mathbf{1}}$ & $\mathbf{h}_{\mathbf{2}}$ & $\mathbf{h}_{\mathbf{3}}$ \\
\hline \hline$\# 1$ & 0 & $-1.28-\mathrm{i}^{*} 0.3$ & $1.61+\mathrm{i}^{*} 2.3$ & $0.17+\mathrm{i}^{*} 0.2$ \\
\hline$\# 2$ & $-1.0-\mathrm{i}^{*} 0.5$ & $0.10+\mathrm{i}^{*} 1.1$ & $1.47+\mathrm{i}^{*} 1.8$ & $-0.48-\mathrm{i}^{*} 0.5$ \\
\hline$\# 3$ & 0 & $-0.28+\mathrm{i}^{*} 0.5$ & $0.37-\mathrm{i}^{*} 1.0$ & $0.04-\mathrm{i}^{*} 0.1$ \\
\hline$\# 4$ & $-0.2+\mathrm{i}^{*} 0.4$ & $0.03-\mathrm{i}^{*} 0.2$ & $0.33-\mathrm{i}^{*} 0.8$ & $-0.10+\mathrm{i}^{*} 0.2$ \\
\hline
\end{tabular}

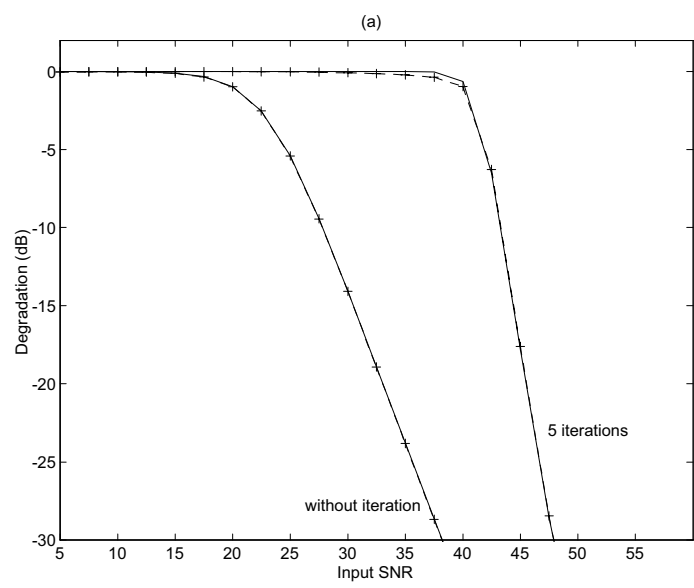

Fig. 2. The degradation factor versus the input SNR (dB). Solid curves are the analysis results and the dash curves with ' + ' are the simulation results of the MSC-based $\mathrm{BCE}$.

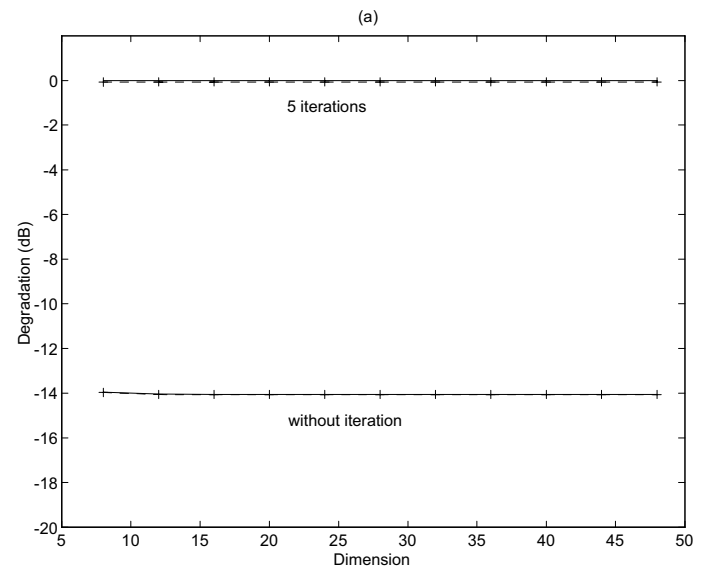

Fig. 3. The degradation factor versus the dimension. Solid curves are the analysis results and the dash curves with ' + ' are the simulation results of the MSC-based $\mathrm{BCE}$.

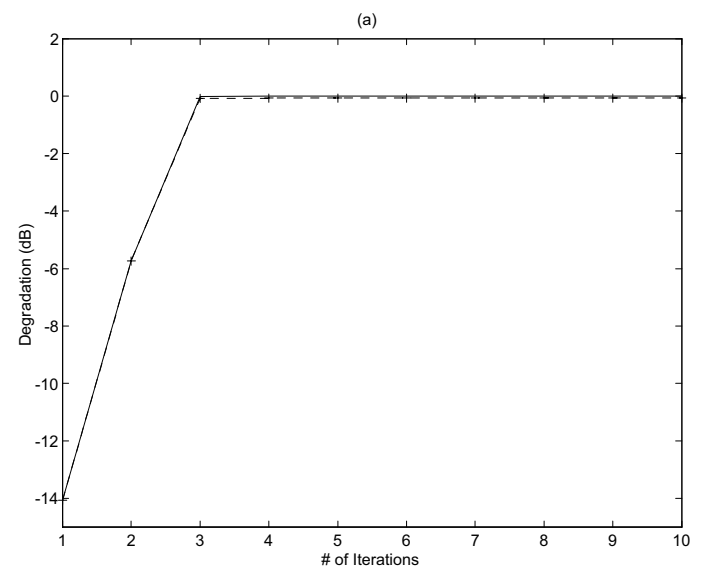

Fig. 4. The degradation factor versus the number of iterations. Solid curves are the analysis results and the dash curves with '+' are the simulation results of the MSC-based $\mathrm{BCE}$. 